\title{
Self-reported sleep apnoea and mortality in patients from the Swedish Obese Subjects study
}

\author{
N.S. Marshall*, L. Delling" , R.R. Grunstein*, M. Peltonen ${ }^{*}$, C.D. Sjöström\#, \\ K. Karason" ${ }^{\#}$ L.M.S. Carlsson ${ }^{\#}$, J. Hedner ${ }^{+}$, K. Stenlöf ${ }^{\#}$ and L. Sjöström ${ }^{\#}$
}

ABSTRACT: Sleep apnoea is associated with increased mortality in sleep clinic and community population groups. It is unclear whether a clinical report of sleep apnoea results in additional mortality risk in patients with severe obesity.

The Swedish Obese Subjects (SOS) study is a nonrandomised controlled trial of bariatric surgery versus conventional treatment for the treatment of severe obesity and its complications (mean \pm SD body mass index $41 \pm 5 \mathrm{~kg} \cdot \mathrm{m}^{-2}$ ). The presence or absence of sleep apnoea (witnessed pauses in breathing) was determined by self-reporting at baseline in 3,953 patients who were observed for 54,236 person-yrs (mean 13.5 maximum 21.0 yrs).

Sleep apnoea was reported by 934 (23.6\%) patients at baseline and was a significant univariate predictor of mortality (hazard ratio $(95 \% \mathrm{Cl}) 1.74(1.40-2.18))$. In a range of multivariate models of mortality risk, controlling for $\leqslant \mathbf{1 6}$ other potential confounders and established mortality risk factors, sleep apnoea remained a significant prognostic factor (fully adjusted model 1.29 (1.01-1.65)).

Self-reported sleep apnoea is an independent prognostic marker of all-cause mortality in obese patients.

KEYWORDS: Mortality, obesity, sleep apnoea

$\mathbf{T}$ here is increasing evidence that sleep apnoea is a risk factor for mortality independent of obesity. This has been shown both in clinical settings [1-5] and, more recently, in three community-based cohorts [6-8]. In addition, bariatric surgery is an effective treatment for sleep apnoea [9].

Severe obesity (body mass index (BMI) $>37 \mathrm{~kg} \cdot \mathrm{m}^{-2}$ ) is strongly associated with mortality $[10,11]$. One of the mechanisms might be sleep apnoea, which is very common in severe obesity and causes impaired cardiovascular function [7, 8, 12, 13], and also impairs psycho-social health [14]. However, it is unclear whether sleep apnoea still confers a higher mortality risk in patients, who all have severe obesity. In addition, there remain lingering doubts about the possible role of visceral obesity being the real cause of at least some of the mortality attributed to obstructive sleep apnoea (OSA) [6, 15]. Studying the OSA-mortality association in people who are all severely obese should help clarify both whether this effect can be seen in the severely obese and whether the population-wide effects of sleep apnoea are in addition to the other established effects of obesity on health.
In the Swedish Obese Subjects (SOS) study, bariatric surgery was associated with a reduced overall mortality compared with conventional treatment in matched, obese controls (hazard ratio 0.71) [11]. Patients in the SOS study have now been followed for $\leqslant 22$ yrs and are well characterised for health risk factors [11,16-18]. Our aim here was to investigate whether self-reported sleep apnoea is independently associated with increased mortality. An additional question posed here is whether remission of apnoea at 2 yrs in patients originally reporting sleep apnoea at baseline is predictive of lower mortality compared with patients in whom that apnoea persists at 2 yrs.

\section{METHODS}

The SOS study is a prospective, controlled intervention trial of bariatric surgery versus conventional treatment for the treatment of severe obesity and its complications. The study design and primary findings are described in detail elsewhere [11, 16-18]. Briefly, 2,010 obese patients (BMI $>34 \mathrm{~kg} \cdot \mathrm{m}^{-2}$ for males and $>38 \mathrm{~kg} \cdot \mathrm{m}^{-2}$ for females, aged $37-60$ yrs at baseline) in the surgically treated group received one of the following three surgical procedures: gastric bypass, gastric banding or
AFFILIATIONS

*National Health and Medical Research Council Centre for Integrated Research and Understanding of Sleep (CIRUS), Woolcock Institute of Medical Research, Sydney School of Medicine, University of Sydney, Sydney, Australia

\# Institute of Medicine, Sahlgrenska Academy, Gothenburg University,

${ }^{+}$Dept of Pulmonary Medicine and Allergology, Sahlgrenska University Hospital, Gothenburg, Sweden.

'Diabetes Prevention Unit, National Institute of Health and Welfare, Helsinki, Finland.

CORRESPONDENCE

N.S. Marshall

Sleep and Circadian Research Group Woolcock Institute of Medical

Research

P0 Box M77

Missenden Road

NSW 2050

Australia

E-mail: nmarshall@woolcock.org.au

Received:

Feb 062011

Accepted after revision:

May 012011

First published online:

May 262011 
vertical-banded gastroplasty [19]. An additional 2,037 patients received nonsurgical treatment for obesity, which was nonstandardised and was handled according the prevailing custom in the unit where the patient was enrolled. Anti-obesity drugs were not available in Sweden until 1998. The primary outcome of this trial was mortality during a mean 11-yr follow-up [11].

Randomisation to gastric surgery was considered to be unethical in 1987 by six out of the seven relevant institutional review boards due to the high mortality associated with these procedures at that time. Gastric surgery patients were, however, matched by a computer algorithm [16] with control patients. The following variables were considered when constructing the computer program to run the matching algorithm: age, sex, weight, height, waist and hip circumferences, systolic pressure, serum cholesterol, serum triglyceride levels, smoking status, diabetes, menopause, four psycho-social variables with mortality associations, and two treatment preference variables indicative of personality traits. Exclusion criteria have been listed in detail [17]; however, patients were not excluded for hypertension, lipid disturbance, diabetes, history of myocardial infarction or stroke, or for any sleep disorder or sleep disturbance.

\section{Examinations}

Patients were recruited from 480 primary health care centres in Sweden. Standard application forms were sent to 11,453 subjects between September 1987 and November 2000. Of these, 8,966 patients met age and height-weight requirements. Information was provided about the surgical and medical treatments and patients were asked for their preference on treatment. Of the 7,593 patients who returned these questionnaires, 6,905 completed a registry examination.

Patients who became candidates for surgical intervention also underwent a surgical examination. These took place, on average, 8 months after the registry examination and 5 months before surgery.

Matched controls were selected by computer algorithm 8 weeks before surgery, and both the surgical candidate and control attended separate examinations 4 weeks before the surgical intervention and thus the start date for the trial. Subsequent examination occurred at $0.5,1.0,2.0,3.0,4.0,6.0,8.0$ and $10.0 \mathrm{yrs}$ after surgery for both of the matched patient groups.

\section{Assessments}

Anthropometry, biochemical variables and blood pressure were measured at baseline, and at 2 and 10 yrs. Anthropometric measurements were obtained as described elsewhere [16, 17], blood samples were taken in the morning after a 10-12-h fast and were analysed in the accredited Central Laboratory of the Sahlgrenska University Hospital, Gothenburg, Sweden (accredited according to European Standard 45001; www. european-accreditation.org). Blood pressure was ascertained via a sphygmomanometer placed on the right arm after $15 \mathrm{~min}$ of supine rest.

\section{Criteria for health and disease}

Diabetes was defined as a fasting blood glucose level of $\geqslant 6.1 \mathrm{mmol} \cdot \mathrm{L}^{-1}$ or a patient report of using a glucose-lowering agent. Hypertension was defined as a systolic blood pressure of $\geqslant 140 \mathrm{mmHg}$ and/or a diastolic blood pressure $\geqslant 90 \mathrm{mmHg}$, or treatment with antihypertensive medication. A baseline questionnaire included questions about previous myocardial infarction, stroke and cancer.

In the SOS study, five questions about sleep apnoea were asked. The patients were asked if they had a regular home partner and if the partner had observed pauses in breathing during sleep. They also answered questions about daytime sleepiness, irresistible napping, and loud and disruptive snoring. The last three questions were to be answered on a five-point scale: never, rarely, sometimes, often and very often.

In these analyses, sleep apnoea was a binary variable assessed by asking all patients: "Has anyone told you that you have brief breathing pauses during your sleep?" (translated from Swedish).

\section{Ascertainment of mortality and vitality}

The Swedish Population Register and Address Register (SPAR) is cross-matched with the SOS database using social security numbers once a year on November 1 . In our analyses, SPAR was cross-checked until November 1, 2008.

\section{Data analyses}

Baseline characteristics were investigated for their association with sleep apnoea using the Chi-squared, unpaired t-test or

\begin{tabular}{|c|c|c|c|}
\hline $\begin{array}{l}\text { Baseline } \mathrm{C} \\
\text { with and } \mathrm{V}\end{array}$ & $\begin{array}{l}\text { haracteristics } \\
\text { ithout apnoea }\end{array}$ & $\begin{array}{l}\text { of males and fel } \\
\text { at baseline }\end{array}$ & males \\
\hline & Sleep apnoea & No sleep apnoea & p-value \\
\hline Subjects n & 934 & 3019 & \\
\hline Age yrs & $48.1 \pm 6.0$ & $47.9 \pm 6.2$ & 0.54 \\
\hline Females & $460(49.3)$ & $2349(77.8)$ & $<0.001$ \\
\hline Surgery & $493(52.8)$ & $1471(48.7)$ & 0.03 \\
\hline BMI $\mathrm{kg} \cdot \mathrm{m}^{-2}$ & $41.3 \pm 4.8$ & $41.2 \pm 4.7$ & 0.55 \\
\hline Weight kg & $122.7 \pm 18.1$ & $116.4 \pm 16.1$ & $<0.001$ \\
\hline Weight loss at 2 yrs $\mathbf{k g}$ & $-16.4 \pm 19.3$ & $-14.7 \pm 18.4$ & $0.053^{\#}$ \\
\hline Waist/hip ratio & $1.01 \pm 0.07$ & $0.98 \pm 0.08$ & $<0.001$ \\
\hline Waist circumference $\mathrm{cm}$ & $125.8 \pm 11.3$ & $122.1 \pm 11.4$ & $<0.001$ \\
\hline Sagittal diameter $\mathbf{c m}$ & $29.1 \pm 3.9$ & $27.8 \pm 3.6$ & $<0.001$ \\
\hline $\begin{array}{l}\text { Average blood pressure } \\
\qquad \mathrm{mmHg}\end{array}$ & $116.3 \pm 14.0$ & $114.0 \pm 13.7$ & $<0.001$ \\
\hline Total cholesterol $\mathrm{mmol} \cdot \mathrm{L}^{-1}$ & $5.8 \pm 1.1$ & $5.7 \pm 1.1$ & 0.02 \\
\hline HDL cholesterol $\mathrm{mmol} \cdot \mathrm{L}^{-1}$ & $1.13 \pm 0.27$ & $1.21 \pm 0.29$ & $<0.001$ \\
\hline Triglycerides $\mathrm{mmol} \cdot \mathrm{L}^{-1}$ & $2.0(1.5-2.8)$ & $1.75(1.29-2.40)$ & $<0.001^{\#}$ \\
\hline Glucose $\mathrm{mmol} \cdot \mathrm{L}^{-1}$ & $4.8(4.3-5.7)$ & $4.7(4.2-5.4)$ & $<0.001^{\#}$ \\
\hline Insulin $\mathrm{mU} \cdot \mathrm{L}^{-1}$ & $19.2(13.5-27.4)$ & $16.3(11.6-23.4)$ & $<0.001^{\#}$ \\
\hline Diabetes & $189(20.2)$ & $467(15.5)$ & $<0.001$ \\
\hline History of stroke & $14(1.5)$ & $23(0.8)$ & 0.04 \\
\hline History of CVD & $38(4.1)$ & $53(1.8)$ & $<0.001$ \\
\hline History of cancer & $16(1.7)$ & $28(0.9)$ & 0.046 \\
\hline Smokers & $260(27.8)$ & $660(21.8)$ & $<0.001$ \\
\hline
\end{tabular}


TABLE 2 Univariate baseline risk factors for mortality

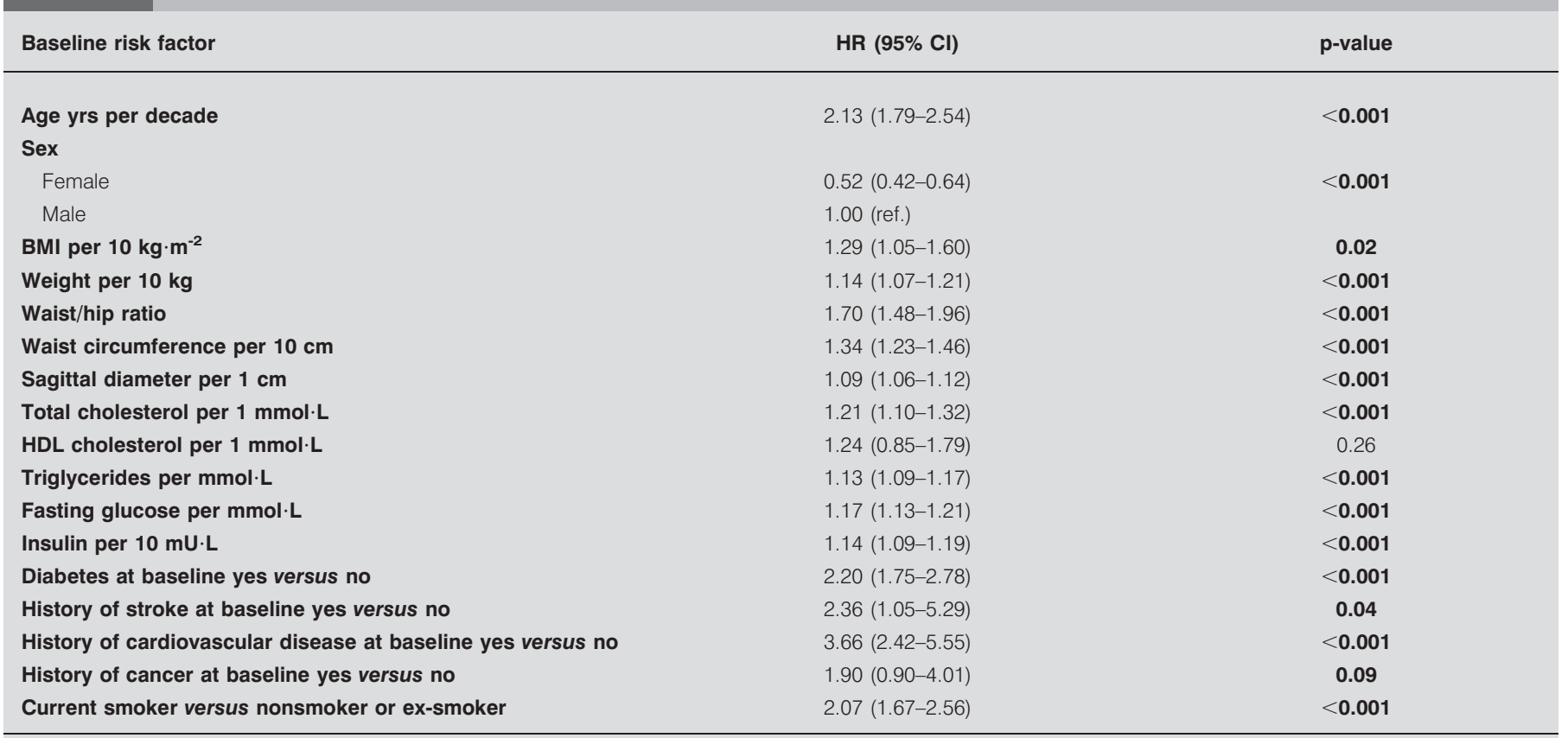

Data are derived from univariate Cox models based on information gathered at baseline pooled across the surgery and control groups. Values in bold are considered to be statistically significant. HR: hazard ratio; BMI: body mass index; HDL: high-density lipoprotein; ref.: reference category.

Wilcoxon tests where appropriate. Univariate associations between baseline characteristics and mortality were investigated with Kaplan-Meier estimates and log-rank tests, and with univariate Cox proportional hazards models.

Multivariate models for mortality were built using Cox regression models, and confirmed by best-subset variable selection. The following variables were forced a priori into the models because of known associations with OSA or mortality: age, sex, obesity, smoking status, blood pressure, total cholesterol, highdensity lipoprotein (HDL) cholesterol and glucose. In addition, other risk factors were examined for independent association with mortality when they exhibited some evidence of a univariate association with either mortality or with sleep apnoea at statistical significance level of $p=0.05$. Schoenfeld and Martingale residuals were used to confirm the proportional hazards and linearity assumptions, respectively.

We further evaluated whether bariatric surgery modified the association between OSA and mortality. This was performed by including an interaction term between sleep apnoea and treatment group in our main models. In addition, Cox models were used to evaluate whether regression of sleep apnoea from baseline to the 2-yr follow-up was associated with better survival than continued report of sleep apnoea after 2 yrs. Analyses were undertaken by N.S. Marshall using SAS (v 9.2; SAS Institute Inc., Cary, NC, USA).

\section{RESULTS}

A total of 4,047 patients were enrolled in the study (2,010 in the surgical arm and 2,037 in the control arm). Information about apnoea status at baseline was supplied by 3,953 (97.7\%) subjects.

Baseline characteristics for the pooled control and surgical intervention groups across sleep apnoea categories are described in table 1. Differences between the two groups are described elsewhere [11, 18]. The mean observation time was $13.9 \mathrm{yrs}$ (maximum $21.0 \mathrm{yrs}$ ) in those without sleep apnoea and $13.2 \mathrm{yrs}$ (maximum $21.0 \mathrm{yrs}$ ) in the sleep apnoea group. There were 237

\section{TABLE 3 Association of sleep apnoea with mortality}

\begin{tabular}{|c|c|c|c|}
\hline Sleep apnoea status & Unadjusted HR (95\% Cl) & Partially adjusted HR $(95 \% \mathrm{Cl})^{\#}$ & Fully adjusted $\mathrm{HR}(95 \% \mathrm{Cl})$ \\
\hline Sleep apnoea & $1.74(1.40-2.18)$ & $1.30(1.02-1.67)$ & $1.29(1.01-1.65)$ \\
\hline No sleep apnoea & 1.00 (ref.) & 1.00 (ref.) & 1.00 (ref.) \\
\hline
\end{tabular}

The partially adjusted model was built only from those variables that were statistically significant in the full model. HR: hazard ratio; ref.: reference category. ${ }^{\#}$ : adjusted for baseline age, sex, treatment group (surgical versus control), waist/hip ratio, sagittal diameter, smoking status (current versus never- or ex-smoker), high-density lipoprotein cholesterol, glucose, and baseline history of cardiovascular disease (yes versus no); ${ }^{`}$ : additionally adjusted for baseline history of stroke (yes versus no), diabetes (yes versus no), triglycerides, mean blood pressure, insulin, history of cancer (yes versus no) and total cholesterol. 


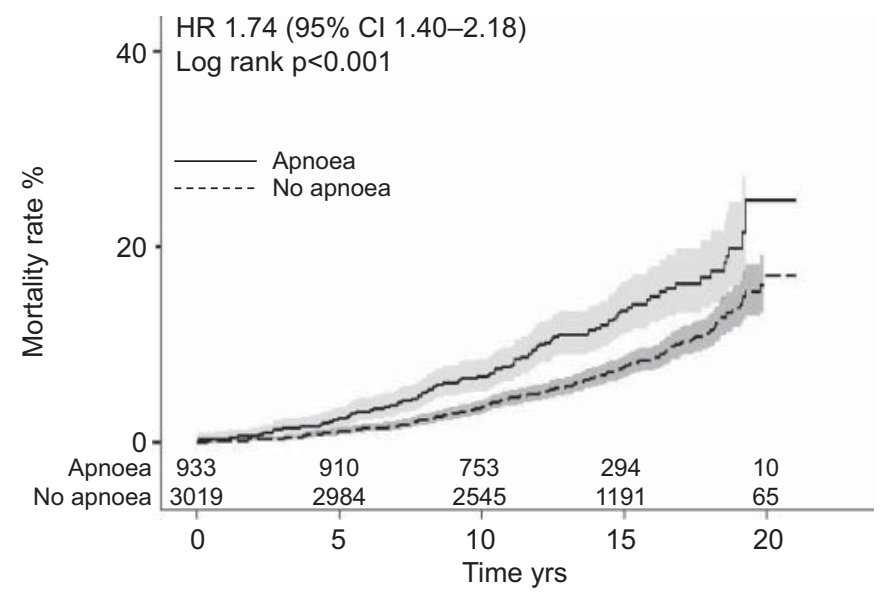

FIGURE 1. Univariate association between the presence or absence of sleep apnoea reported at baseline and 21-yr mortality in the Swedish Obese Subjects study pooled across both surgical intervention and control groups. Numbers across the bottom of the graph indicate the numbers of patients being observed at each 5-yr time-point. HR: hazard ratio.

deaths observed in those without sleep apnoea (raw mortality $7.9 \%)$ and 117 (12.5\%) in those with sleep apnoea.

Univariate mortality risk factors, other than apnoea status, are detailed in table 2. Self-reported sleep apnoea was associated with excess mortality at both a univariate level and in subsequent multivariate proportional hazards models (table 3 ). The significant univariate association between apnoea and mortality is further demonstrated in a Kaplan-Meier plot (fig. 1). We also ran models where all deaths within the first 2 yrs were excluded and these were also statistically significant.

Several definitions of blood pressure were evaluated, including mean arterial pressure, but average blood pressure $(0.5 \times$ systolic $+0.5 \times$ diastolic) gave the best fit to observed mortality. The best fit for body habitus to mortality was the combined variation in sagittal diameter and waist/hip ratio. We also tested BMI and neck circumference as linear and categorical variables.

We attempted to test whether bariatric surgery might mitigate the OSA-mortality association by stratifying by treatment group. Sleep apnoea did elevate mortality risk in the control group (partially adjusted HR 1.41 (95\% CI 1.01-1.97) and fully adjusted HR 1.36 (0.96-1.91)). But this elevation was not as evident in the surgical group (partially adjusted HR 1.17 (0.821.69) and fully adjusted HR 1.14 (0.79-1.63); table 4). We attempted to verify whether this apparent difference was significant but, in the full model (table 3), the introduction of an interaction between treatment group and sleep apnoea was not statistically significant.

\section{Secondary subgroup analyses}

In people with sleep apnoea at baseline who were alive at 2 yrs and reported their sleep apnoea status (349 females and 330 males), there was a significant survival advantage in those females whose sleep apnoea had regressed ( $n=203 ; p=0.04)$ (fig. 2). This univariate association in females was not significant after adjustment for age. Males whose sleep apnoea had regressed at 2 yrs did not have a greater survival advantage as compared with those without remission $(n=202 ; p=0.5)($ fig. 3$)$.

\section{DISCUSSION}

Self-reported sleep apnoea appears to be an independent prognostic marker of all-cause mortality in patients in the SOS study. This confirms previous observations in both populationbased cohorts and in cardiovascular and sleep clinic cohorts that sleep apnoea is a mortality risk factor $[8,15]$. Thus, even amongst the severely obese, patient reports of sleep apnoea are not incidental.

Bariatric surgery in severely obese patients has benefits that extend beyond the correction of apnoea [11, 16, 18, 20]; our findings could also have implied that patients with sleep apnoea might represent an important subgroup, like diabetics [21], where bariatric surgery is especially effective [22]. Our direct statistical testing of this hypothesis, however, was not significant.

The mortality risk associated with sleep apnoea in the SOS study was lower (HR 1.29) than the risk associated with sleep apnoea in the general community ( $\mathrm{HR}>2.0)$ [6-8]. It is possible that the lower estimate here could be explained by a misclassification of patients' apnoea status caused by inaccurate selfreports. SHARKEY et al. [23], for instance, found that self-reported apnoeas in females presenting for bariatric surgery did correlate with sleep apnoea ascertained via an overnight sleep study, but with notable misclassification of people with OSA saying they

TABLE 4 Mortality risk associated with sleep apnoea in the surgical and the control groups separately

\begin{tabular}{|c|c|c|c|}
\hline Sleep apnoea status & Unadjusted HR (95\% Cl) & Partially adjusted HR $(95 \% \mathrm{Cl})^{\#}$ & Fully adjusted HR (95 \\
\hline \multicolumn{4}{|l|}{ Control } \\
\hline Sleep apnoea & $1.87(1.38-2.54)$ & $1.41(1.01-1.97)$ & $1.36(0.96-1.91)$ \\
\hline No sleep apnoea & 1.00 (ref.) & 1.00 (ref.) & 1.00 (ref.) \\
\hline \multicolumn{4}{|l|}{ Surgical } \\
\hline Sleep apnoea & $1.64(1.19-2.27)$ & $1.17(0.82-1.69)$ & $1.14(0.79-1.63)$ \\
\hline No sleep apnoea & 1.00 (ref.) & 1.00 (ref.) & 1.00 (ref.) \\
\hline
\end{tabular}

HR: hazard ratio; ref.: reference category. ${ }^{\#}$ : adjusted for baseline age, sex, waist/hip ratio, sagittal diameter, smoking status (current versus never- or ex-smoker), highdensity lipoprotein cholesterol, glucose, history of cardiovascular disease (yes versus no); ": additionally adjusted for baseline history of stroke (yes versus no), diabetes (yes versus no), triglycerides, mean blood pressure, insulin, history of cancer (yes versus no), and total cholesterol. 


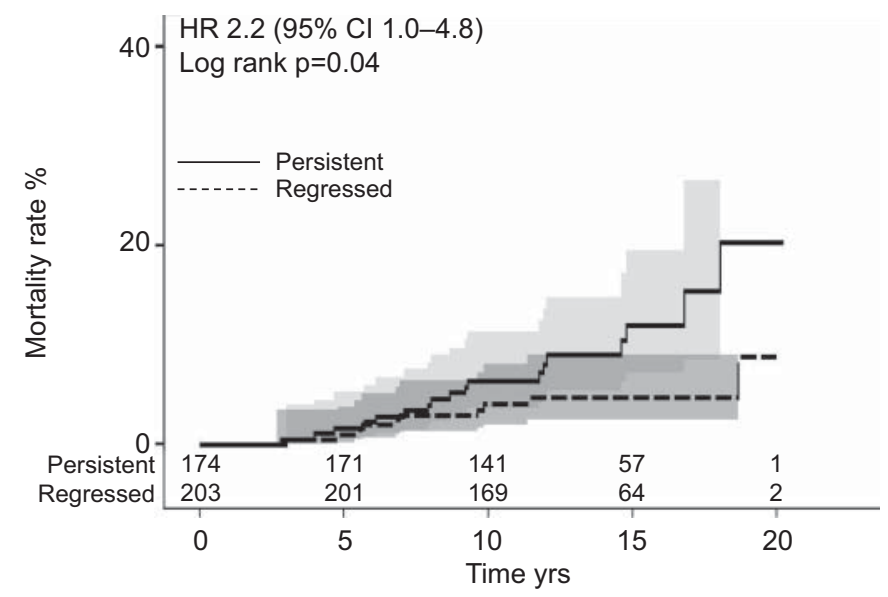

FIGURE 2. Univariate mortality rates in female patients with sleep apnoea at baseline who reported that their sleep apnoea had either persisted or regressed at 2 yrs. Numbers across the bottom of the graph indicate the numbers of patients being observed at each 5-yr time-point. HR: hazard ratio.

did not have the condition. However, our small HR may also be an indication that the risk attributable to sleep apnoea decreases as obesity and associated risk factors worsen. It has also been suggested that some of the mortality risk associated with sleep apnoea might be attributable to the residual confounding effects on the metabolism of visceral obesity [15]. The severe obesity in the SOS study coupled with our specific control of central obesity markers (sagittal diameter and waist/hip ratio) should have controlled for some of this potential residual confounding. Previous studies have indicated that sleep apnoea may not be a mortality risk for females in the same way it is for males [8]. The predominantly female make-up of the SOS cohort ( $\sim 70 \%)$ may thus partially explain our relatively lower than expected magnitude of risk.

Excess weight is the major modifiable risk factor for sleep apnoea [24, 25]. In community-based cohorts, incident sleep apnoea is more often observed in those gaining weight than

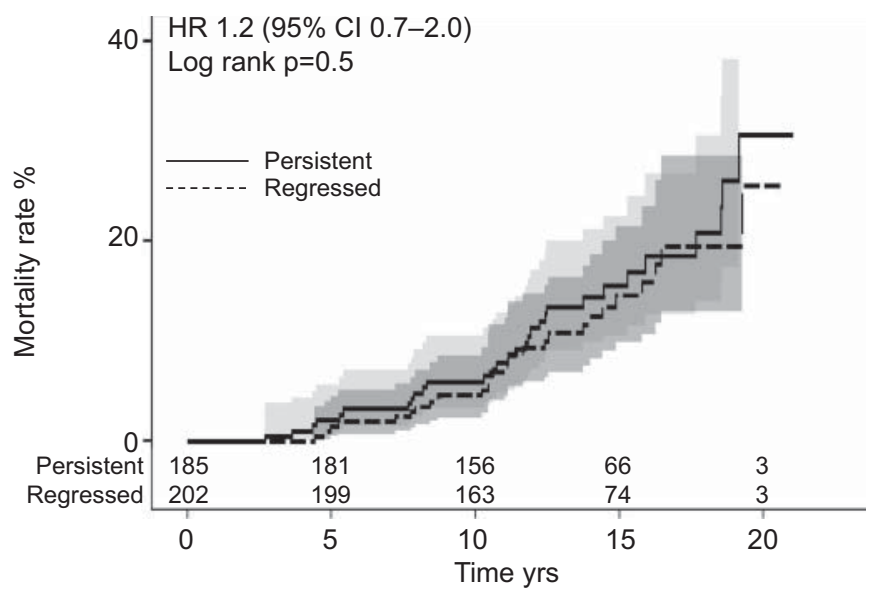

FIGURE 3. Univariate mortality rates in male patients with sleep apnoea at baseline who reported that their sleep apnoea had either persisted or regressed at 2 yrs. Numbers across the bottom of the graph indicate the numbers of patients being observed at each 5-yr time-point. HR: hazard ratio. those with stable weight [26-28]. Obesity reduction in OSA patients reduces sleep apnoea severity, whether weight loss is achieved via pharmacotherapy [29], diet [30-32] or surgery [20]. As a result of this, the relative weakness of the mortality association, in addition to the already-mentioned misclassification problem, may also be explained by the success of the surgical interventions in reducing body mass. We tested this hypothesis by stratifying by treatment group across all our models. In the parsimonious model, sleep apnoea was an independent predictor of mortality in the control group (HR 1.4; $p=0.04$ ) but not in the surgical group (HR 1.2; $p=0.4)$ (table 4). This indirectly suggested that the marked weight loss caused by bariatric surgery resulted in subsequent regression of sleep apnoea [24], a phenomenon which we have already demonstrated in this cohort [9]. We also analysed whether the regression of sleep apnoea at 2 yrs was associated with greater survival in those with sleep apnoea at baseline. Apnoea regression was associated with significantly greater survival compared with persistent sleep apnoea in females, but not in males ( $p=0.04$ and $p=0.5$ for females and males, respectively). However, in females, this association was not significant after controlling for age $(p=0.09)$. Combined with the lack of a population-based cohort that has definitively linked sleep apnoea to female mortality [6-8], these secondary analyses in a relatively smaller number of patients should be interpreted with caution, given the likelihood of misclassification. However, the SOS cohort suggests that treating sleep apnoea in severely obese females may extend life expectancy. Sleep apnoea is poorly studied in females and thus our observation may present a valuable clinical trial target.

There are a number of strengths and limitations in using the SOS cohort to study sleep apnoea and mortality risk. The patients were severely obese at baseline and therefore had a high prior likelihood of having sleep apnoea. Due to the successful intervention (weight loss surgery) in this study, we were able to observe both a cohort of people with high and stable body mass (the control group) alongside an equally wellcharacterised cohort of people who have lost substantial body mass (the surgical group). The female predominance of the cohort may be important, as is the ascertainment of sleep apnoea through self-reports. The self-report of sleep apnoea via reported breathing pauses during sleep is unlikely to be sensitive to the presence of sleep apnoea. For instance, a recent study of female bariatric patients indicated that self-reported apnoea was $\sim 58 \%$ sensitive and $88 \%$ specific to detecting at least mild OSA, with $93 \%$ of patients reporting witnessed apnoeas actually testing positive for sleep apnoea on an overnight sleep study (true positive rate) [23]. We therefore suspect that, in the SOS cohort, there is a substantial proportion of the apnoea-negative group who really did have sleep apnoea. Previous studies have used self-reporting of sleep apnoea or snoring and also found, for instance, an increased mortality risk in sleepy snorers [33]. Nevertheless, the interventional SOS cohort contains almost 4,000 severely obese patients followed for $\leqslant 22$ yrs and is, from this perspective, not currently matched anywhere else in the sleep literature.

Data from the SOS study confirm that the higher mortality observed in people with sleep apnoea both in the general community [6-8] and in cohorts of sleep apnoea patients [1-5] is also present in patients with severe obesity. Although the 
magnitude of this added risk appears attenuated when compared with these previous reports, self-reported sleep apnoea in severely obese patients does provide additional information about mortality risk.

\section{SUPPORT STATEMENT}

The Swedish Obese Subjects study was supported by grants from Hoffman-La Roche, AstraZeneca, Cederroth, Ethicon, Sanofi-Adventis and the Swedish Medical Research Council (to L. Sjöström), and NHMRC grants 352483, 457-94 and 264598 to RR. Grunstein.

\section{STATEMENT OF INTEREST}

Statements of interest for C.D. Sjöström, L.M.S. Carlsson and for the study itself can be found at www.erj.ersjournals.com/site/misc/ statements.xhtml

\section{REFERENCES}

1 Campos-Rodriguez F, Pena-Grinan N, Reyes-Nunez N, et al. Mortality in obstructive sleep apnea-hypopnea patients treated with positive airway pressure. Chest 2005; 128: 624-633.

2 He J, Kryger M, Zorick F, et al. Mortality and apnea index in obstructive sleep apnea: experience in 385 male patients. Chest 1988; 94: 9-14.

3 Lavie P, Herer P, Peled R, et al. Mortality in sleep apnea patients a multivariate analysis of risk factors. Sleep 1995; 18: 149-157.

4 Marin J, Carrizo S, Vicente E, et al. Long-term cardiovascular outcomes in men with obstructive sleep apnoea-hypopnoea with or without treatment with continuous positive airway pressure: an observational study. Lancet 2005; 365: 1046-1053.

5 Yaggi HK, Concato J, Kernan WN, et al. Obstructive sleep apnea as a risk factor for stroke and death. N Engl J Med 2005; 353: 2034-2041.

6 Marshall NS, Wong KKH, Liu PY, et al. Sleep apnea as an independent risk factor for all-cause mortality: the Busselton Health Study. Sleep 2008; 31: 1079-1085.

7 Young T, Finn L, Peppard PE, et al. Sleep disordered breathing and mortality: eighteen-year follow-up of the Wisconsin sleep cohort. Sleep 2008; 31: 1071-1078.

8 Punjabi NM, Caffo BS, Goodwin JL, et al. Sleep-disordered breathing and mortality: a prospective cohort study. PLoS Med 2009; 6: e1000132.

9 Grunstein R, Stenlof K, Hedner J, et al. Two year reduction in sleep apnea symptoms and associated diabetes incidence after weight loss in severe obesity. Sleep 2007; 30: 703-710.

10 Finkelstein EA, Brown DS, Wrage LA, et al. Individual and aggregate years-of-life-lost associated with overweight and obesity. Obesity 2009; 18: 333-339.

11 Sjöström L, Narbro K, Sjöström CD, et al. Effects of bariatric surgery on mortality in Swedish obese subjects. N Engl J Med 2007; 357: 741-752.

12 Barbe F, Duran-Cantolla J, Capote F, et al. Long-term effect of continuous positive airway pressure in hypertensive patients with sleep apnea. Am J Respir Crit Care Med, 181: 718-726.

13 Haentjens P, Van Meerhaeghe A, Moscariello A, et al. The impact of continuous positive airway pressure on blood pressure in patients with obstructive sleep apnea syndrome: evidence from a meta-analysis of placebo-controlled randomized trials. Arch Intern Med 2007; 167: 757-764.
14 Grunstein R, Stenlof K, Hedner J, et al. Impact of self-reported sleep-breathing disturbances on psychosocial performance in the Swedish Obese Subjects (SOS) study. Sleep 1995; 18: 635-643.

15 Pack AI, Platt AB, Pien G. Does untreated obstructive sleep apnea lead to death? Sleep 2008; 31: 1067-1068.

16 Sjöström L, Gummesson A, Sjöström CD, et al. Effects of bariatric surgery on cancer incidence in obese patients in Sweden (Swedish Obese Subjects study): a prospective, controlled intervention trial. Lancet Oncol 2009; 10: 653-662.

17 Sjöström L, Larsson B, Backman L, et al. Swedish Obese Subjects (SOS): recruitment for an intervention study and a selected description of the obese state. Int J Obes Relat Metab Disord 1992; 16: 465-479.

18 Sjöström L, Lindroos A-K, Peltonen M, et al. Lifestyle, diabetes, and cardiovascular risk factors 10 years after bariatric surgery. N Engl J Med 2004; 351: 2683-2693.

19 Sjöström L. Surgical intervention as a strategy for treatment of obesity. Endocrine 2000; 13: 213-230.

20 Buchwald H, Avidor Y, Braunwald E, et al. Bariatric surgery: a systematic review and meta-analysis. JAMA 2004; 292: 1724-1737.

21 Dixon JB, O'Brien PE, Playfair J, et al. Adjustable gastric banding and conventional therapy for type 2 diabetes: a randomized controlled trial. JAMA 2008; 299: 316-323.

22 Dixon JB, Schachter LM, O'Brien PE. Polysomnography before and after weight loss in obese patients with severe sleep apnea. Int $J$ Obes Relat Metab Disord 2005; 29: 1048-1054.

23 Sharkey KM, Machan JT, Tosi C, et al. Predicting obstructive sleep apnea among women candidates for bariatric surgery. J Womens Health 2010; 19: 1833-1841.

24 Young T, Peppard PE, Taheri S. Excess weight and sleepdisordered breathing. J Appl Physiol 2005; 99: 1592-1599.

25 Marshall NS, Grunstein RR. Losing weight in moderate to severe obstructive sleep apnoea. BMJ 2009; 339: b4363.

26 Newman AB, Foster G, Givelber R, et al. Progression and regression of sleep-disordered breathing with changes in weight: the Sleep Heart Health Study. Arch Intern Med 2005; 165: 2408-2413.

27 Peppard PE, Young T, Palta M, et al. Longitudinal study of moderate weight change and sleep disordered breathing. JAMA 2000; 284: 3015-3021.

28 Tishler P, Larkin E, Schlucter M, et al. Incidence of sleepdisordered breathing in an urban adult population. JAMA 2003; 289: 2230-2237.

29 Yee BJ, Phillips CL, Banerjee D, et al. The effect of sibutramineassisted weight loss in men with obstructive sleep apnoea. Int J Obes 2007; 31: 161-168.

30 Tuomilehto HPI, Seppa JM, Partinen MM, et al. Lifestyle intervention with weight reduction: first-line treatment in mild obstructive sleep apnea. Am J Respir Crit Care Med 2009; 179: 320-327.

31 Foster GD, Borradaile KE, Sanders MH, et al. A randomized study on the effect of weight loss on obstructive sleep apnea among obese patients with type 2 diabetes: the Sleep AHEAD Study. Arch Intern Med 2009; 169: 1619-1626.

32 Johansson K, Neovius M, Lagerros YT, et al. Effect of a very low energy diet on moderate and severe obstructive sleep apnoea in obese men: a randomised controlled trial. BMJ 2009; 339: 1365-1370.

33 Lindberg E, Janson C, Svardsudd K, et al. Increased mortality among sleepy snorers: a prospective population based study. Thorax 1998; 53: 631-637. 